\title{
Fidelity is a sub-martingale for discrete-time quantum filters
}

\author{
Pierre Rouchon*
}

March 2011

\begin{abstract}
Fidelity is known to increase through any Kraus map: the fidelity between two density matrices is less than the fidelity between their images via a Kraus map. We prove here that, in average, fidelity is also increasing for discrete-time quantum filters attached to an arbitrary Kraus map: fidelity between the density matrix of the underlying Markov chain and the density matrix of the associated quantum filter is a sub-martingale. This result is not restricted to pure states. It also holds true for mixed states.
\end{abstract}

\section{Introduction}

Quantum filtering was developed, in its modern theoretical form, by Belavkin during the 1980's [1] (see also [2] for a recent tutorial introduction). Quantum filtering proposes a theory for statistical inference strongly inspired from quantum optical systems. These systems are described by continuous-time quantum stochastic differential equations (stochastic master equations). The stability of the obtained quantum filters, i.e, the fact that quantum filters are asymptotically independent of their initial states, has been investigated in several papers. In [9] sufficient convergence conditions are established: they are related to observability issues. ${ }^{1}$ In [4] convergence of the estimate to the physical state is addressed for a generic Hamiltonian and measurement operator: the analysis relies on the fact that, for pure states, the fidelity between the real state and its estimate is proved to be a sub-martingale.

Quantum filtering is not restricted to continuous time. It can also be used for discretetime systems as in [5] where a quantum filter is used inside a state-feedback scheme. This note proves that fidelity between the estimate and real state is always a sub-martingale

\footnotetext{
*Mines ParisTech: pierre.rouchon@mines-paristech.fr

${ }^{1}$ See [10] for general connections between the stability of nonlinear filters and mathematical systems theory concepts such as stability, observability and detectability.
} 
for such discrete-time quantum filters. This does not mean that quantum filters are always convergent. Nevertheless, this sub-martingale combined with stochastic invariance arguments could be useful to prove convergence in specific situations.

Fidelity $F(\sigma, \rho)$ between two quantum states described by density matrices $\rho$ and $\sigma$ coincides with their Frobenius inner product $\operatorname{Tr}(\rho \sigma)$ when at least one of two density matrices $\rho$ or $\sigma$ is a projector of rank one, i.e. a pure state. ${ }^{2}$ The fact that fidelity is a sub martingale for pure states is a known fact. For continuous-time systems, the convergence investigations proposed in [4] relies on this fact. For discrete-time systems, theorem 3 in [7], based only on Cauchy-Schwartz inequalities, proves that the Frobenius inner product between the estimate and real state is always a sub-martingale whatever the purity of the estimate and real state are. Consequently, if we assume that the real state remains pure, then, fidelity coincides with Frobenius inner product and thus is known to be a sub-martingale. When the real state and its estimate are mixed, fidelity does not coincide with Frobenius inner product and the proof that it is a sub-martingale does not result directly from published papers, as far as we know. Fidelity between the arbitrary states $\rho$ and $\sigma$ is given by $\operatorname{Tr}^{2}(\sqrt{\sqrt{\rho} \sigma \sqrt{\rho}})$, an expression much more difficult to manipulate than $\operatorname{Tr}(\rho \sigma)$. The contribution of this note (theorems 1 and 2 relying on inequalities (4) and (5), respectively) consists in proving that, in the discrete-time case and for arbitrary purity, the fidelity between the estimate and real state is sub-martingale.

In section 2, we recall the Kraus map associated to any quantum channel, the associated Markov chain (quantum Monte-Carlo trajectories) and highlight the basic inequalities (4) and (5) underlying theorems 1 and 2 proved in the remaining sections. In section 3, we consider the quantum filter attached to a quantum trajectory and prove theorem 1: fidelity is a sub-martingale. In section 4 we extend this result to a family of Markov chains attached to the same Kraus map. In section 5, we conclude by noting that, contrarily to fidelity, trace distance and relative entropy are not always super-martingales.

The author thanks Mazyar Mirrahimi and Ramon Van Handel for fruitful comments and interesting references.

\section{Kraus maps and quantum Markov chains}

Take the Hilbert space $S=\mathbb{C}^{n}$ of dimension $n>0$ and consider a quantum channel described by the Kraus map (see [8], chapter 8 or [6], chapter 4)

$$
\mathcal{K}(\rho)=\sum_{\mu=1}^{m} M_{\mu} \rho M_{\mu}^{\dagger}
$$

where

\footnotetext{
${ }^{2}$ There are two closely related definitions of the fidelity. The "mathematical" definition used, e.g., in [8] corresponds to the square root of the "physical" definition we have adopted in this paper for simplicity sakes.
} 
- $\rho$ is the density matrix describing the input quantum state, $\mathcal{K}(\rho)$ being then the output quantum state; $\rho \in \mathbb{C}^{n \times n}$ is a density matrix, i.e., an Hermitian matrix semipositive definite and of trace one;

- for each $\mu \in\{1, \ldots, m\}, M_{\mu} \in \mathbb{C}^{n \times n} /\{0\}$, and $\sum_{\mu} M_{\mu}^{\dagger} M_{\mu}=I$.

To this quantum channel is associated the following discrete-time Markov chain (quantum Monte-Carlo trajectories, see, e.g., [6]):

$$
\rho_{k+1}=\mathcal{M}_{\mu_{k}}\left(\rho_{k}\right)
$$

where

- $\rho_{k}$ is the quantum state at sampling time $t_{k}$ and $k$ the sampling index $\left(t_{k}<t_{k+1}\right)$.

- $\mu_{k} \in\{1, \ldots, m\}$ is a random variable; $\mu_{k}=\mu$ with probability $p_{\mu}\left(\rho_{k}\right)=\operatorname{Tr}\left(M_{\mu} \rho_{k} M_{\mu}^{\dagger}\right)$.

- $\mathcal{M}_{\mu}(\rho)=\frac{1}{\operatorname{Tr}\left(M_{\mu} \rho M_{\mu}^{\dagger}\right)} M_{\mu} \rho M_{\mu}^{\dagger}=\frac{1}{p_{\mu}(\rho)} M_{\mu} \rho M_{\mu}^{\dagger}$.

Quantum probabilities derive from density matrices which are dual (via the Frobenius Hermitian product $\operatorname{Tr}\left(A B^{\dagger}\right), A, B, n \times n$ matrices with complex entries) to the set of Hermitian $n \times n$ matrices (space of observables). The Kraus map (1) leads to a generalized observable (or effect valued measure) $\mathcal{P} \mapsto \sum_{\mu \in \mathcal{P}} M_{\mu}^{\dagger} M_{\mu}$ where $\mathcal{P}$ is any subset of $\{1, \ldots, m\}$. The map $(\mathcal{P}, \rho) \mapsto \sum_{\mu \in \mathcal{P}} M_{\mu} \rho M_{\mu}^{\dagger}$ is known as Davies instrument (see chapter 4 of [3]). It formalizes a measurement notion relating the state (the density matrix $\rho$ ), measure properties (here $\mathcal{P}$ living in the $\sigma$-algebra generated by the singletons of the discrete set $\{1, \ldots, m\})$ and an output state conditional on the observed values (here $\sum_{\mu \in \mathcal{P}} M_{\mu} \rho M_{\mu}^{\dagger}$ up to a normalization to ensure a unit trace). The non-linear operators $\mathcal{M}_{\mu}$ just define the one-step transition mechanism for the above quantum Markov chain. Such setting can be seen as an appropriate analogue of what one encounters in classical discrete-time filtering problems.

Fidelity is one of several ways to measure difference between density matrices and is specific to quantum theory. The Kraus map tends to increase fidelity $F$ (see [8], theorem 9.6, page 414): for all density matrices $\rho$ and $\sigma$, one has

$$
\operatorname{Tr}^{2}(\sqrt{\sqrt{\mathcal{K}(\sigma)} \mathcal{K}(\rho) \sqrt{\mathcal{K}(\sigma)}})=F(\mathcal{K}(\sigma), \mathcal{K}(\rho)) \geq F(\sigma, \rho)=\operatorname{Tr}^{2}(\sqrt{\sqrt{\sigma} \rho \sqrt{\sigma}})
$$

where, for any Hermitian semi-positive matrix $A=U \Lambda U^{\dagger}, U$ unitary matrix and $\Lambda=$ $\operatorname{diag}\left\{\lambda_{l}\right\}_{l \in\{1, \ldots, n\}}, \sqrt{A}=U \sqrt{\Lambda} U^{\dagger}$ with $\sqrt{\Lambda}=\operatorname{diag}\left\{\sqrt{\lambda_{l}}\right\}_{l \in\{1, \ldots, n\}}$.

The conditional expectation of $\rho_{k+1}$ knowing $\rho_{k}$ is given by the Kraus map:

$$
\mathbb{E}\left(\rho_{k+1} / \rho_{k}\right)=\mathcal{K}\left(\rho_{k}\right) .
$$


This result from the trivial identity $\sum_{\mu=1}^{m} \operatorname{Tr}\left(M_{\mu} \rho M_{\mu}^{\dagger}\right) \frac{M_{\mu} \rho M_{\mu}^{\dagger}}{\operatorname{Tr}\left(M_{\mu} \rho M_{\mu}^{\dagger}\right)},=\mathcal{K}(\rho)$. In section 3, we show during the proof of theorem (1) the following inequality

$$
\sum_{\mu=1}^{m} \operatorname{Tr}\left(M_{\mu} \rho M_{\mu}^{\dagger}\right) F\left(\frac{M_{\mu} \sigma M_{\mu}^{\dagger}}{\operatorname{Tr}\left(M_{\mu} \sigma M_{\mu}^{\dagger}\right)}, \frac{M_{\mu} \rho M_{\mu}^{\dagger}}{\operatorname{Tr}\left(M_{\mu} \rho M_{\mu}^{\dagger}\right)}\right) \geq F(\sigma, \rho)
$$

for any density matrices $\rho$ and $\sigma$. The left-hand side is related to a conditional expectation. Inequality (4), attached to the probabilistic mapping (2), can be seen as the stochastic counter-part of inequality (3) attached to the deterministic mapping (1). When for some $\mu, \operatorname{Tr}\left(M_{\mu} \sigma M_{\mu}^{\dagger}\right)=0$ with $\operatorname{Tr}\left(M_{\mu} \rho M_{\mu}^{\dagger}\right)>0$, the $\mu$-term in the sum (4) is not defined. This is not problematic, since in this case, if we replace $\frac{M_{\mu} \sigma M_{\mu}^{\dagger}}{\operatorname{Tr}\left(M_{\mu} \sigma M_{\mu}^{\dagger}\right)}$ by $\frac{M_{\mu} \xi M_{\mu}^{\dagger}}{\operatorname{Tr}\left(M_{\mu} \xi M_{\mu}^{\dagger}\right)}$ where $\xi$ is any density matrix such that $\operatorname{Tr}\left(M_{\mu} \xi M_{\mu}^{\dagger}\right)>0$, this term is then well defined (in a multi-valued way) and inequality (4) remains satisfied for any such $\xi$.

During the proof of theorem (8), we extend this inequality to any partition of $\{1, \ldots, m\}$ into $p \geq 1$ sub-sets $\mathcal{P}_{\nu}$ :

$$
\sum_{\nu=1}^{\nu=p} \operatorname{Tr}\left(\sum_{\mu \in \mathcal{P}_{\nu}} M_{\mu} \rho M_{\mu}^{\dagger}\right) F\left(\frac{\sum_{\mu \in \mathcal{P}_{\nu}} M_{\mu} \sigma M_{\mu}^{\dagger}}{\operatorname{Tr}\left(\sum_{\mu \in \mathcal{P}_{\nu}} M_{\mu} \sigma M_{\mu}^{\dagger}\right)}, \frac{\sum_{\mu \in \mathcal{P}_{\nu}} M_{\mu} \rho M_{\mu}^{\dagger}}{\operatorname{Tr}\left(\sum_{\mu \in \mathcal{P}_{\nu}} M_{\mu} \rho M_{\mu}^{\dagger}\right)}\right) \geq F(\sigma, \rho)
$$

\section{The standard case.}

Take a realization of the Markov chain associated to the Kraus map $\mathcal{K}$. Assume that we detect, for each $k$, the jump $\mu_{k}$ but that we do not know the initial state $\rho_{0}$. The objective is to propose at sampling $k$, an estimation $\hat{\rho}_{k}$ of $\rho_{k}$ based on the past detections $\mu_{0}, \ldots, \mu_{k-1}$. The simplest method consists in starting from an initial estimation $\hat{\rho}_{0}$ and at each sampling step to jump according to the detection. This leads to the following estimation scheme known as a quantum filter (see, e.g., [11]):

$$
\hat{\rho}_{k+1}=\mathcal{M}_{\mu_{k}}\left(\hat{\rho}_{k}\right)
$$

with $p_{\mu}\left(\rho_{k}\right)=\operatorname{Tr}\left(M_{\mu} \rho_{k} M_{\mu}\right)$ as probability of $\mu_{k}=\mu$. Notice that when $\operatorname{Tr}\left(M_{\mu_{k}} \hat{\rho}_{k} M_{\mu_{k}}\right)=$ $0, \mathcal{M}_{\mu_{k}}\left(\hat{\rho}_{k}\right)$ is not defined and should be replaced by $\mathcal{M}_{\mu_{k}}(\xi)$ where $\xi$ is any density matrix such that $\operatorname{Tr}\left(M_{\mu_{k}} \hat{\xi} M_{\mu_{k}}\right)>0$ (take, e.g., $\left.\xi=\frac{1}{n} I_{d}\right)$. The theorem here below could be useful to investigate the convergence of $\hat{\rho}_{k}$ towards $\rho_{k}$ as $k$ increases.

Theorem 1. Consider the Markov chain of state $\left(\rho_{k}, \hat{\rho}_{k}\right)$ satisfying (2) and (6). Then $F\left(\hat{\rho}_{k}, \rho_{k}\right)$ is a sub-martingale: $\mathbb{E}\left(F\left(\hat{\rho}_{k+1}, \rho_{k+1}\right) /\left(\hat{\rho}_{k}, \rho_{k}\right)\right) \geq F\left(\hat{\rho}_{k}, \rho_{k}\right)$.

The proof proposed here below deals with the general case when both $\rho_{k}$ and $\hat{\rho}_{k}$ can be mixed states. It relies on arguments similar to those used for the proof of theorem 9.6 in [8]. They are based on a kind of lifting process, usual in differential geometry. They consist in introducing additional variables and embedding the Hilbert space $S$ into the tensor product 
with a copy $Q$ of $S$ and with the environment-model space $E$. In the "augmented space" $S \otimes Q \otimes E$, the quantum dynamics (2) read as an unitary transformation followed by an orthogonal projection. At sampling step $k$, the estimated and real states are lifted to the "augmented space" via a standard procedure known as purification. These purifications are then subject to an unitary transformation followed by the same projection. Partial traces over $Q \otimes E$ provide then the estimated and real states at sampling time $k+1$. At each sampling step $k$, the purifications of the estimated and real states are chosen according to Ulhmann's theorem: the square of modulus of their Hermitian product is maximum and coincides thus with the fidelity. The remaining part of the proof is based on simple manipulations of Hermitian products and Cauchy-Schwartz inequalities.

Proof. The density matrices $\rho$ and $\hat{\rho}$ are operators from $S=\mathbb{C}^{n}$ to $S$. Take a copy $Q=\mathbb{C}^{n}$ of $S$ and consider the composite system living on $S \otimes Q \equiv \mathbb{C}^{n^{2}}$. Then $\hat{\rho}$ and $\rho$ correspond to partial traces versus $Q$ of projectors $|\hat{\psi}\rangle\langle\hat{\psi}|$ and $|\psi\rangle\langle\psi|$ associated to pure states $|\hat{\psi}\rangle$ and $|\psi\rangle \in S \otimes Q:$

$$
\hat{\rho}=\operatorname{Tr}_{Q}(|\hat{\psi}\rangle\langle\hat{\psi}|), \quad \rho=\operatorname{Tr}_{Q}(|\psi\rangle\langle\psi|)
$$

$|\hat{\psi}\rangle$ and $|\psi\rangle$ are called purifications of $\hat{\rho}$ and $\rho$. They are not unique but one can always choose them such that $F(\hat{\rho}, \rho)=|\langle\hat{\psi} \mid \psi\rangle|^{2}$ (Uhlmann's theorem, see [8], theorem 9.4 page 410). Denote by $\left|\hat{\psi}_{k}\right\rangle$ and $\left|\psi_{k}\right\rangle$ such purifications of $\hat{\rho}_{k}$ and $\rho_{k}$ satisfying

$$
F\left(\hat{\rho}_{k}, \rho_{k}\right)=\left|\left\langle\hat{\psi}_{k} \mid \psi_{k}\right\rangle\right|^{2}
$$

We have

$$
\mathbb{E}\left(F\left(\hat{\rho}_{k+1}, \rho_{k+1}\right) /\left(\hat{\rho}_{k}, \rho_{k}\right)\right)=\sum_{\mu=1}^{m} p_{\mu}\left(\rho_{k}\right) F\left(\mathcal{M}_{\mu}\left(\hat{\rho}_{k}\right), \mathcal{M}_{\mu}\left(\rho_{k}\right)\right)
$$

The matrices $\mathcal{M}_{\mu}\left(\hat{\rho}_{k}\right)$ and $\mathcal{M}_{\mu}\left(\rho_{k}\right)$ are also density matrices.

We will consider now the space $S \otimes Q \otimes E$ where $E=\mathbb{C}^{m}$ is the Hilbert space of the environment appearing in the system-environment model of the Kraus map (1) (see [6], chapter 4 entitled "The environment is watching"). Thus exist $\left|e_{0}\right\rangle$ a unitary element of $E$, an ortho-normal basis $(|\mu\rangle)_{\mu \in\{1, \ldots, m\}}$ of $E$ and a unitary transformation $U$ (not unique) on $S \otimes E$ such that, for all $|\phi\rangle \in S$,

$$
U\left(|\phi\rangle \otimes\left|e_{0}\right\rangle\right)=\sum_{\mu=1}^{m}\left(M_{\mu}|\phi\rangle\right) \otimes|\mu\rangle .
$$

For each $\mu$, denote by $P_{\mu}$ the orthogonal projector onto the subspace $S \otimes(\mathbb{C}|\mu\rangle)$. Then $P_{\mu} U\left(|\phi\rangle \otimes\left|e_{0}\right\rangle\right)=\left(M_{\mu}|\phi\rangle\right) \otimes|\mu\rangle$ and $\sum_{\mu} P_{\mu}=I$. For any density matrix $\rho$ on $S$,

$$
P_{\mu} U\left(\rho \otimes\left|e_{0}\right\rangle\left\langle e_{0}\right|\right) U^{\dagger} P_{\mu}=M_{\mu} \rho M_{\mu}^{\dagger} \otimes|\mu\rangle\langle\mu|
$$

and thus

$$
\operatorname{Tr}_{E}\left(P_{\mu} U\left(\rho \otimes\left|e_{0}\right\rangle\left\langle e_{0}\right|\right) U^{\dagger} P_{\mu}\right)=M_{\mu} \rho M_{\mu}^{\dagger} .
$$


The unitary transformation $U$ of $S \otimes E$ can be extended to $S \otimes Q \otimes E \equiv S \otimes E \otimes Q$ by setting $V=U \otimes I$ ( $I$ is identity on $Q$ ). Since $\left|\psi_{k}\right\rangle \in S \otimes Q$ is a purification of $\rho_{k}$, we have

$$
M_{\mu} \rho_{k} M_{\mu}^{\dagger}=\operatorname{Tr}_{E \otimes Q}\left(P_{\mu} V\left(\left|\psi_{k}\right\rangle\left\langle\psi_{k}|\otimes| e_{0}\right\rangle\left\langle e_{0}\right|\right) V^{\dagger} P_{\mu}\right) .
$$

Set $\left|\phi_{k}\right\rangle=\left|\psi_{k}\right\rangle \otimes\left|e_{0}\right\rangle \in S \otimes Q \otimes E$ and $\left|\chi_{k}\right\rangle=V\left|\phi_{k}\right\rangle$. Using $P_{\mu}^{2}=P_{\mu}$, we have

$$
p_{\mu}\left(\rho_{k}\right)=\operatorname{Tr}\left(M_{\mu} \rho_{k} M_{\mu}^{\dagger}\right)=\left\langle\phi_{k}\left|V^{\dagger} P_{\mu} V\right| \phi_{k}\right\rangle=\| P_{\mu}\left|\chi_{k}\right\rangle \|^{2} .
$$

Thus, for each $\mu$, the state $\left|\chi_{k \mu}\right\rangle=\frac{1}{\sqrt{p_{\mu}\left(\rho_{k}\right)}} P_{\mu}\left|\chi_{k}\right\rangle$ is a purification of $\mathcal{M}_{\mu}\left(\rho_{k}\right)$ :

$$
\mathcal{M}_{\mu}\left(\rho_{k}\right)=\operatorname{Tr}_{Q \otimes E}\left(\left|\chi_{k \mu}\right\rangle\left\langle\chi_{k \mu}\right|\right) .
$$

Similarly set $\left|\hat{\phi}_{k}\right\rangle=\left|\hat{\psi}_{k}\right\rangle \otimes\left|e_{0}\right\rangle$ and $\left|\hat{\chi}_{k}\right\rangle=V\left|\hat{\phi}_{k}\right\rangle$. For each $\mu,\left|\hat{\chi}_{k \mu}\right\rangle=\frac{1}{\sqrt{p_{\mu}\left(\hat{\rho}_{k}\right)}} P_{\mu}\left|\hat{\chi}_{k}\right\rangle$ is also a purification of $\mathcal{M}_{\mu}\left(\hat{\rho}_{k}\right)$. By Uhlmann's theorem,

$$
F\left(\mathcal{M}_{\mu}\left(\hat{\rho}_{k}\right), \mathcal{M}_{\mu}\left(\rho_{k}\right)\right) \geq\left|\left\langle\hat{\chi}_{k \mu} \mid \chi_{k \mu}\right\rangle\right|^{2} .
$$

Thus we have

$$
\mathbb{E}\left(F\left(\hat{\rho}_{k+1}, \rho_{k+1}\right) /\left(\hat{\rho}_{k}, \rho_{k}\right)\right) \geq \sum_{\mu=1}^{m} p_{\mu}\left(\rho_{k}\right)\left|\left\langle\hat{\chi}_{k \mu} \mid \chi_{k \mu}\right\rangle\right|^{2} .
$$

Since $V$ is unitary,

$$
\left|\left\langle\hat{\chi}_{k} \mid \chi_{k}\right\rangle\right|^{2}=\left|\left\langle\hat{\phi}_{k} \mid \phi_{k}\right\rangle\right|^{2}=\left|\left\langle\hat{\psi}_{k} \mid \psi_{k}\right\rangle\right|^{2}=F\left(\hat{\rho}_{k}, \rho_{k}\right) .
$$

Let us show that $\sum_{\mu=1}^{m} p_{\mu}\left(\rho_{k}\right)\left|\left\langle\hat{\chi}_{k \mu} \mid \chi_{k \mu}\right\rangle\right|^{2} \geq\left|\left\langle\hat{\chi}_{k} \mid \chi_{k}\right\rangle\right|^{2}$. We have

$$
p_{\mu}\left(\rho_{k}\right)\left|\left\langle\hat{\chi}_{k \mu} \mid \chi_{k \mu}\right\rangle\right|^{2}=\left|\left\langle\hat{\chi}_{k \mu} \mid P_{\mu} \chi_{k}\right\rangle\right|^{2}=\left|\left\langle\hat{\chi}_{k \mu} \mid \chi_{k}\right\rangle\right|^{2},
$$

thus it is enough to prove that $\sum_{\mu=1}^{m}\left|\left\langle\hat{\chi}_{k \mu} \mid \chi_{k}\right\rangle\right|^{2} \geq\left|\left\langle\hat{\chi}_{k} \mid \chi_{k}\right\rangle\right|^{2}$. Denote by $\hat{R} \subset S \otimes Q \otimes E$ the vector space spanned by the ortho-normal basis $\left(\left|\hat{\chi}_{k \mu}\right\rangle\right)_{\mu \in\{1, \ldots, m\}}$ and by $\hat{P}$ the projector on $\hat{R}$. Since

$$
\left|\hat{\chi}_{k}\right\rangle=\sum_{\mu=1}^{m} P_{\mu}\left|\hat{\chi}_{k}\right\rangle=\sum_{\mu=1}^{m} \sqrt{p_{\mu}\left(\hat{\rho}_{k}\right)}\left|\hat{\chi}_{k \mu}\right\rangle
$$

$\left|\hat{\chi}_{k}\right\rangle$ belongs to $\hat{R}$ and thus $\left|\left\langle\hat{\chi}_{k} \mid \chi_{k}\right\rangle\right|^{2}=\left.\left|\left\langle\hat{\chi}_{k}|\hat{P}| \chi_{k}\right\rangle\right\rangle\right|^{2}$. We conclude by Cauchy-Schwartz inequality

$$
\left|\left\langle\hat{\chi}_{k} \mid \chi_{k}\right\rangle\right|^{2}=\left.\left|\left\langle\hat{\chi}_{k}|\hat{P}| \chi_{k}\right\rangle\right\rangle\right|^{2} \leq\left\|\hat{\chi}_{k}\right\|^{2} \| \hat{P}\left|\chi_{k}\right\rangle\left\|^{2}=\right\| \hat{P}\left|\chi_{k}\right\rangle \|^{2}=\sum_{\mu=1}^{m}\left|\left\langle\hat{\chi}_{k \mu} \mid \chi_{k}\right\rangle\right|^{2} .
$$




\section{The coarse-grained case.}

Let us consider another Markov chain associated to a partition of $\{1, \ldots, m\}$ into $p \geq 1$ sub-sets $\mathcal{P}_{\nu}$ (aggregation of several quantum jumps via "partial Kraus maps"):

$$
\rho_{k+1}=\frac{1}{\operatorname{Tr}\left(\sum_{\mu \in \mathcal{P}_{\nu_{k}}} M_{\mu} \rho_{k} M_{\mu}^{\dagger}\right)}\left(\sum_{\mu \in \mathcal{P}_{\nu_{k}}} M_{\mu} \rho_{k} M_{\mu}^{\dagger}\right)
$$

where $\nu_{k}=\nu$ with probability $\operatorname{Tr}\left(\sum_{\mu \in \mathcal{P}_{\nu}} M_{\mu} \rho_{k} M_{\mu}^{\dagger}\right)$. It is clear that $\mathbb{E}\left(\rho_{k+1} / \rho_{k}\right)=\mathcal{K}\left(\rho_{k}\right)$ : the Markov chains (2) and (7) are attached to the same Kraus map (1). Consider the associated quantum filter

$$
\hat{\rho}_{k+1}=\frac{1}{\operatorname{Tr}\left(\sum_{\mu \in \mathcal{P}_{\nu_{k}}} M_{\mu} \hat{\rho}_{k} M_{\mu}^{\dagger}\right)}\left(\sum_{\mu \in \mathcal{P}_{\nu_{k}}} M_{\mu} \hat{\rho}_{k} M_{\mu}^{\dagger}\right)
$$

where the jump index $\nu_{k}$ coincides with the jump index $\nu_{k}$ in (7). Then we have the following theorem.

Theorem 2. Consider the Markov chain of state $\left(\rho_{k}, \hat{\rho}_{k}\right)$ satisfying (7) and (8). Then $F\left(\hat{\rho}_{k}, \rho_{k}\right)$ is a sub-martingale: $\mathbb{E}\left(F\left(\hat{\rho}_{k+1}, \rho_{k+1}\right) /\left(\hat{\rho}_{k}, \rho_{k}\right)\right) \geq F\left(\hat{\rho}_{k}, \rho_{k}\right)$.

Proof. It is similar to the proof of theorem 1. We will just point out here the main changes using the same notations. We start from

$$
\mathbb{E}\left(F\left(\hat{\rho}_{k+1}, \rho_{k+1}\right) /\left(\hat{\rho}_{k}, \rho_{k}\right)\right)=\sum_{\nu=1}^{p} \tilde{p}_{\nu}\left(\rho_{k}\right) F\left(\tilde{\mathcal{M}}_{\nu}\left(\hat{\rho}_{k}\right), \tilde{\mathcal{M}}_{\nu}\left(\rho_{k}\right)\right) .
$$

where we have set

$$
\tilde{p}_{\nu}(\rho)=\operatorname{Tr}\left(\sum_{\mu \in \mathcal{P}_{\nu}} M_{\mu} \rho M_{\mu}^{\dagger}\right), \quad \tilde{\mathcal{M}}_{\nu}(\rho)=\frac{1}{\tilde{p}_{\nu}(\rho)}\left(\sum_{\mu \in \mathcal{P}_{\nu}} M_{\mu} \rho M_{\mu}^{\dagger}\right) .
$$

With $\tilde{P}_{\nu}$ the orthogonal projector on $S \otimes Q \otimes \operatorname{span}\left\{|\mu\rangle, \mu \in \mathcal{P}_{\nu}\right\}$ and $\tilde{M}_{\nu}(\rho)=\sum_{\mu \in \mathcal{P}_{\nu}} M_{\mu} \rho M_{\mu}^{\dagger}$, we have

$$
\sum_{\mu \in \mathcal{P}_{\nu}} M_{\mu} \hat{\rho}_{k} M_{\mu}^{\dagger}=\operatorname{Tr}_{Q \otimes E}\left(\tilde{P}_{\nu} V\left(\left|\psi_{k}\right\rangle\left\langle\psi_{k}|\otimes| e_{0}\right\rangle\left\langle e_{0}\right|\right) V^{\dagger} \tilde{P}_{\nu}\right)
$$

and

$$
\tilde{p}_{\nu}\left(\rho_{k}\right)=\operatorname{Tr}\left(\sum_{\mu \in \mathcal{P}_{\nu}} M_{\mu} \hat{\rho}_{k} M_{\mu}^{\dagger}\right)=\left\langle\phi_{k}\left|V^{\dagger} \tilde{P}_{\nu} V\right| \phi_{k}\right\rangle=\| \tilde{P}_{\nu}\left|\chi_{k}\right\rangle \|^{2}
$$

For each $\nu$, the state $\left|\tilde{\chi}_{k \nu}\right\rangle=\frac{1}{\sqrt{\tilde{p}_{\nu}\left(\rho_{k}\right)}} \tilde{P}_{\nu}\left|\chi_{k}\right\rangle$ is a purification of $\tilde{\mathcal{M}}_{\nu}\left(\rho_{k}\right)$ :

$$
\tilde{\mathcal{M}}_{\nu}\left(\rho_{k}\right)=\operatorname{Tr}_{Q \otimes E}\left(\left|\tilde{\chi}_{k \nu}\right\rangle\left\langle\tilde{\chi}_{k \nu}\right|\right)
$$


Similarly $\left|\hat{\tilde{\chi}}_{k \nu}\right\rangle=\frac{1}{\sqrt{\tilde{p}_{\nu}\left(\hat{\rho}_{k}\right)}} \tilde{P}_{\nu}\left|\hat{\chi}_{k}\right\rangle$ is also a purification of $\tilde{\mathcal{M}}_{\nu}\left(\hat{\rho}_{k}\right)$. By Uhlmann's theorem,

$$
F\left(\tilde{\mathcal{M}}_{\nu}\left(\hat{\rho}_{k}\right), \tilde{\mathcal{M}}_{\nu}\left(\rho_{k}\right)\right) \geq\left|\left\langle\hat{\tilde{\chi}}_{k \nu} \mid \tilde{\chi}_{k \nu}\right\rangle\right|^{2}
$$

Thus we have

$$
\mathbb{E}\left(F\left(\hat{\rho}_{k+1}, \rho_{k+1}\right) /\left(\hat{\rho}_{k}, \rho_{k}\right)\right) \geq \sum_{\nu=1}^{p} \tilde{p}_{\nu}\left(\rho_{k}\right)\left|\left\langle\hat{\tilde{\chi}}_{k \mu} \mid \tilde{\chi}_{k \mu}\right\rangle\right|^{2} .
$$

Let us show that $\sum_{\nu=1}^{p} \tilde{p}_{\nu}\left(\rho_{k}\right)\left|\left\langle\hat{\tilde{\chi}}_{k \nu} \mid \tilde{\chi}_{k \nu}\right\rangle\right|^{2} \geq\left|\left\langle\hat{\chi}_{k} \mid \chi_{k}\right\rangle\right|^{2}=F\left(\hat{\rho}_{k}, \rho_{k}\right)$. We have

$$
\tilde{p}_{\nu}\left(\rho_{k}\right)\left|\left\langle\hat{\tilde{\chi}}_{k \nu} \mid \tilde{\chi}_{k \nu}\right\rangle\right|^{2}=\left|\left\langle\hat{\tilde{\chi}}_{k \nu} \mid \tilde{P}_{\nu} \chi_{k}\right\rangle\right|^{2}=\left|\left\langle\hat{\tilde{\chi}}_{k \nu} \mid \chi_{k}\right\rangle\right|^{2},
$$

thus it is enough to prove that $\sum_{\nu=1}^{p}\left|\left\langle\hat{\tilde{\chi}}_{k \nu} \mid \chi_{k}\right\rangle\right|^{2} \geq\left|\left\langle\hat{\chi}_{k} \mid \chi_{k}\right\rangle\right|^{2}$. Denote by $\hat{\tilde{R}} \subset S \otimes Q \otimes E$ the vector space spanned by the ortho-normal basis $\left(\left|\hat{\tilde{\chi}}_{k \nu}\right\rangle\right)_{\nu \in\{1, \ldots, p\}}$ and by $\hat{\tilde{P}}$ the projector on $\hat{\tilde{R}}$. Since

$$
\left|\hat{\chi}_{k}\right\rangle=\sum_{\nu=1}^{p} \tilde{P}_{\nu}\left|\hat{\chi}_{k}\right\rangle=\sum_{\nu=1}^{p} \sqrt{\tilde{p}_{\nu}\left(\hat{\rho}_{k}\right)}\left|\hat{\tilde{\chi}}_{k \nu}\right\rangle
$$

$\left|\hat{\chi}_{k}\right\rangle$ belongs to $\hat{\tilde{R}}$ and thus $\left|\left\langle\hat{\chi}_{k} \mid \chi_{k}\right\rangle\right|^{2}=\left.\left|\left\langle\hat{\chi}_{k}|\hat{\tilde{P}}| \chi_{k}\right\rangle\right\rangle\right|^{2}$. We conclude by Cauchy-Schwartz inequality

$$
\left|\left\langle\hat{\chi}_{k} \mid \chi_{k}\right\rangle\right|^{2}=\left.\left|\left\langle\hat{\chi}_{k}|\hat{\tilde{P}}| \chi_{k}\right\rangle\right\rangle\right|^{2} \leq\left\|\hat{\chi}_{k}\right\|^{2} \| \hat{\tilde{P}}\left|\chi_{k}\right\rangle\left\|^{2}=\right\| \hat{\tilde{P}}\left|\chi_{k}\right\rangle \|^{2}=\sum_{\nu=1}^{p}\left|\left\langle\hat{\tilde{\chi}}_{k \nu} \mid \chi_{k}\right\rangle\right|^{2} .
$$

\section{Concluding remarks}

Theorems 1 and 2 are still valid if the Kraus operators $M_{\mu}$ depend on $k$. In particular, $F\left(\hat{\rho}_{k}, \rho_{k}\right)$ remains a sub-martingale even if the Kraus operators depend on $\hat{\rho}_{k}$, i.e., in case of feedback. A natural extension will be to prove that fidelity yields also to a sub-martingale in the continuous-time case. The Markov chain (2) is then replaced by a stochastic differential equation for $\rho$ driven by a Poisson or a Wiener process.

Kraus maps are contractions for the trace distance (see [8], theorem 9.2, page 406): for all density matrices $\sigma, \rho$, one has $\operatorname{Tr}(|\mathcal{K}(\sigma)-\mathcal{K}(\rho)|) \leq \operatorname{Tr}(|\sigma-\rho|)$. When $\sigma$ and $\rho$ are pure states (projectors of rank one), $D(\sigma, \rho)=\sqrt{1-F(\sigma, \rho)}$. Consequently inequality (4) yields to

$$
\sum_{\mu=1}^{m} \operatorname{Tr}\left(M_{\mu} \rho M_{\mu}^{\dagger}\right) D\left(\frac{M_{\mu} \sigma M_{\mu}^{\dagger}}{\operatorname{Tr}\left(M_{\mu} \sigma M_{\mu}^{\dagger}\right)}, \frac{M_{\mu} \rho M_{\mu}^{\dagger}}{\operatorname{Tr}\left(M_{\mu} \rho M_{\mu}^{\dagger}\right)}\right) \leq D(\sigma, \rho)
$$

for any pure states $\sigma$ and $\rho$ (use the fact that $[0,1] \ni x \mapsto \sqrt{1-x}$ is decreasing and concave). It is then tempting to conjecture that the above inequality holds true for any 
mixed states $\rho$ and $\sigma$, i.e., that $D\left(\hat{\rho}_{k}, \rho_{k}\right)=\operatorname{Tr}\left(\left|\hat{\rho}_{k}-\rho_{k}\right|\right)$ is a super-martingale of the Markov chain defined by (2) and (6). Unfortunately, this is not true in general as shown by the following counter-example. Take $n=3, m=2$ and

$$
\rho=\left(\begin{array}{ccc}
\frac{1}{2} & 0 & 0 \\
0 & \frac{1}{2} & 0 \\
0 & 0 & 0
\end{array}\right), \quad \sigma=\left(\begin{array}{ccc}
0 & 0 & 0 \\
0 & \frac{1}{2} & 0 \\
0 & 0 & \frac{1}{2}
\end{array}\right), \quad M_{1}=\left(\begin{array}{ccc}
1 & 0 & 0 \\
0 & \frac{1}{\sqrt{2}} & 0 \\
0 & 0 & 0
\end{array}\right), \quad M_{2}=\left(\begin{array}{ccc}
0 & 0 & 0 \\
0 & \frac{1}{\sqrt{2}} & 0 \\
0 & 0 & 1
\end{array}\right) .
$$

Then $\sum_{\mu=1}^{2} \operatorname{Tr}\left(M_{\mu} \rho M_{\mu}^{\dagger}\right) D\left(\frac{M_{\mu} \sigma M_{\mu}^{\dagger}}{\operatorname{Tr}\left(M_{\mu} \sigma M_{\mu}^{\dagger}\right)}, \frac{M_{\mu} \rho M_{\mu}^{\dagger}}{\operatorname{Tr}\left(M_{\mu} \rho M_{\mu}^{\dagger}\right)}\right)=\frac{4}{3}>1=D(\sigma, \rho){ }^{3}$ The same counter example shows that the relative entropy $\operatorname{Tr}\left(\rho_{k} \log \rho_{k}\right)-\operatorname{Tr}\left(\rho_{k} \log \hat{\rho}_{k}\right)$ is not, in general, a super-martingale.

\section{References}

[1] V.P. Belavkin. Quantum stochastic calculus and quantum nonlinear filtering. Journal of Multivariate Analysis, 42(2):171-201, 1992.

[2] L. Bouten, R. van Handel, and M. R. James. An introduction to quantum filtering. SIAM J. Control Optim., 46:2199-2241, 2007.

[3] E.B. Davies. Quantum Theory of Open Systems. Academic Press, 1976.

[4] L. Diosi, Th. Konrad, A. Scherer, and J. Audretsch. Coupled Ito equations of continuous quantum state measurement and estimation. J. Phys. A: Math. Gen., 39:L575L581, 2006.

[5] I. Dotsenko, M. Mirrahimi, M. Brune, S. Haroche, J.-M. Raimond, and P. Rouchon. Quantum feedback by discrete quantum non-demolition measurements: towards ondemand generation of photon-number states. Physical Review A, 80: 013805-013813, 2009.

[6] S. Haroche and J.M. Raimond. Exploring the Quantum: Atoms, Cavities and Photons. Oxford University Press, 2006.

[7] M. Mirrahimi, I. Dotsenko, and P. Rouchon. Feedback generation of quantum fock states by discrete qnd measures. In Decision and Control, 2009 held jointly with the 2009 28th Chinese Control Conference. CDC/CCC 2009. Proceedings of the 48th IEEE Conference on, pages 1451 -1456, 2009.

[8] M.A. Nielsen and I.L. Chuang. Quantum Computation and Quantum Information. Cambridge University Press, 2000.

${ }^{3}$ Notice that we have $\sum_{\mu=1}^{2} \operatorname{Tr}\left(M_{\mu} \rho M_{\mu}^{\dagger}\right) F\left(\frac{M_{\mu} \sigma M_{\mu}^{\dagger}}{\operatorname{Tr}\left(M_{\mu} \sigma M_{\mu}^{\dagger}\right)}, \frac{M_{\mu} \rho M_{\mu}^{\dagger}}{\operatorname{Tr}\left(M_{\mu} \rho M_{\mu}^{\dagger}\right)}\right)=\frac{1}{4}+\frac{1}{12}>\frac{1}{4}=F(\sigma, \rho)$. 
[9] R. van Handel. The stability of quantum Markov filters. Infin. Dimens. Anal. Quantum Probab. Relat. Top., 12:153-172, 2009.

[10] R. van Handel. Nonlinear filtering and systems theory. In 19th International Symposium on Mathematical Theory of Networks and Systems (MTNS 2010), July 2010.

[11] H.M. Wiseman and G.J. Milburn. Quantum Measurement and Control. Cambridge University Press, 2009. 